\title{
Synthesis and characterization of a novel hollow nanoparticle-based SnS crystal product with microcluster-like 3D network hierarchitectures
}

\author{
Xinhua Xu • Chika Takai · Takashi Shirai · Masayoshi Fuji* \\ Advanced Ceramics Research Center, Nagoya Institute of Technology, Honmachi \\ 3-101-1, Tajimi, Gifu 507-0033, Japan
}

\begin{abstract}
:
$\mathrm{SnS}$ is an important semiconductor and its performance depends greatly on its morphology and 3D architecture. So the synthesis for it with specific structures has been widely paid attention. Herein, a novel hollow nanoparticle-based SnS crystal product with microcluster-like 3D network hierarchitectures was developed, via a template-assisted solvothermal route in diethylene glycol (DEG) solvent, followed by annealing in $\mathrm{N}_{2}$ atmosphere and subsequent corrosion of template by dilute acid. $\mathrm{CaCO}_{3}$ nanoparticles were used as the template, $\mathrm{SnCl}_{2}$ as the tin source, thiourea and L-cysteine hydrochloride monohydrate (Cys) as two different sulfur sources for a comparison study. Results show that successful fabrication of the hollow-structured SnS product mentioned above was achieved only in the case of using Cys as the sulfur source, mainly due to much stronger adhesion of Cys on $\mathrm{CaCO}_{3}$ template and microcluster-like stacking patterns of $\mathrm{CaCO}_{3}$ nanoparticles in DEG solvent. This novel 3D hierarchical SnS product owns 1 - 5 times higher specific surface area, unique hollow nanostructure units with rich mesopores inside the shells, and scattering-enhanced absorption in UV-vis light region, as against those previously reported micro-scale tin sulfide 3D hierarchitectures assembled by nanopetals,

*Corresponding author. Fax: (+81) 57224 8109; Tel: (+81) 572248110.

E-mail:fuji@nitech.ac.jp
\end{abstract}


nanosheets, nanorods, nanobelts, etc. So it should have much more extensive and promising applications.

Keywords: SnS; hollow nanoparticle; hierarchitecture; solvothermal.

\section{Introduction}

Tin sulfides like $\mathrm{SnS}, \mathrm{SnS}_{2}$ and $\mathrm{Sn}_{2} \mathrm{~S}_{3}$, as important IV-VI group of semiconductors, have attracted a great deal interest due to high potential applications of their unique optical and electrical properties in diverse fields such as lithium ion batteries [1, 2], solar cells [3], photoconductive cells [4], photodetectors [5], photocatalysts [6], gas sensors [7] and so on. Driven by these promising applications, researchers over the past few decades have focused intensive efforts on developing tin sulfide micro- / nano-structures with various shapes, sizes and dimensions, in order to explore their unprecedented shape- / size-dependent properties and structure-induced enhancement properties.

Up to date, the successfully explored tin sulfide micro- / nano-structures mainly include nanoparticles [5], nanorods [3, 8], nanosheets [9], microflowers [2, 10-12], etc.

Of these structures, the former three nano-scaled tin sulfides usually show many benefits in terms of fast kinetics and high activity in practical applications for their small sizes and large surface area effects, while simultaneously suffer from some shortcomings, for instance, low thermodynamic and chemical stability and thereby short cycle life in harsh conditions, since the high activity of them toward surface reactions can readily accelerate their corrosion, damage and inactivation [2, 13]. Compared to them, the latter micro-scaled tin sulfide microflowers always present porous 3D hierarchical and spherical morphologies assembled by plenty of nano-sized 
building units, e.g. nanopetals [2], nanoplates [11], nanosheets [12], nanorods [14] or nanobelts. Such porous 3D hierarchitectures have been proved to be effective in improving the practical performances of tin sulfide-based devices. For example, the Li-ion batteries fabricated by 3D hierarchical tin sulfide microspheres were found to show larger lithium storage capacity, higher rate capability and better cycling stability [2]. And the tin sulfide photocatalyst consisting of flowerlike 3D hierarchical microstructures were detected to perform much higher photocatalytic activity and longer cycle life [15]. The significant improvement of these 3D hierarchical tin sulfides to the related device performances was widely considered to profit from the combination of their complex assembled microstructures and nano-sized building units $[2,13]$.

Owing to the superior properties of the micro-scaled tin sulfide 3D hierarchitectures than ordinary structures in practical applications, it should be worthwhile to do some innovations on the morphology and assembled structure of their nano-sized building blocks for enlarging the structure-induced enhancement properties. Therefore, the synthesis of them with new and unique hierarchitectures is becoming increasingly attractive. Till now, a variety of synthesis techniques have been employed, including thermal evaporation [16], template method [10], hydrothermal / solvothermal method [11-15], etc. However, to our knowledge, almost no any distinct results have yet been acquired, apart from the previously reported 3D hierarchical tin sulfide microflowers assembled by nanopetals [2], nanoplates [11], nanosheets [12], nanorods [14], nanobelts, etc.

Interestingly, in this work, we developed a unique tin sulfide product, which is pure SnS crystalline phase and comprises many microcluster-like 3D network hierarchitectures built by hollow nanoparticles. As is well known, hollow 
nanoparticles usually possess the following sparked characteristics, e.g. low density, low thermal conductivity, large cavity volume and specific surface area, special optical and electronic properties $[17,18]$. Therefore, it is believed that such novel hollow nanoparticle-based 3D hierarchical SnS crystal product would exhibit outstanding properties in much more extensive application fields, relative to the common microflower-like tin sulfide 3D hierarchitectures assembled with nanopetals / nanoplates / nanosheets / nanorods / nanobelts.

The novel hollow nanoparticle-based SnS crystal product here was fabricated via a predominant three-step route involving: i) synthesis of SnS shells by a facile template-assisted solvothermal coating technique, ii) annealing treatment in $\mathrm{N}_{2}$ atmosphere to improve the SnS crystallinity, and iii) corrosion removal of the template cores by dilute acid. During the first step, $\mathrm{CaCO}_{3}$ nanoparticles were used as the template materials, and the SnS shell coating processes were performed in two separated solvothermal systems, where the same tin source but different sulfur sources were used for a comparison study. By investigating the existence state of $\mathrm{CaCO}_{3}$ template nanoparticles in the solvent medium, and comparing the distinct SnS coating results in the two systems, we proposed a possible mechanism to explain the formation of the novel hollow nanoparticle-based SnS 3D hierarchitecture product. Moreover, we examined the specific surface area, porous nature and optical properties of this product in detail, in order to acquire more information available for exploring its potential applications.

\section{Experimental}

\subsection{Synthesis}

$\mathrm{CaCO}_{3}$ nanoparticles with an average size of $80 \mathrm{~nm}$ (Shiraishi Calcium Co., Ltd.) 
were used as the sacrificial template materials, diethylene glycol (DEG) as the organic solvent medium and tin dichloride $\left(\mathrm{SnCl}_{2}\right)$ as the tin source. Thiourea $\left(\mathrm{CH}_{4} \mathrm{~N}_{2} \mathrm{~S}\right.$, illustrated by Fig. 1(a)) and L-cysteine hydrochloride monohydrate $\left(\mathrm{C}_{3} \mathrm{H}_{10} \mathrm{ClNO}_{3} \mathrm{~S}\right.$, illustrated by Fig. 1(b)) were used as two different sulfur sources. For the sake of brevity, the $\mathrm{C}_{3} \mathrm{H}_{10} \mathrm{ClNO}_{3} \mathrm{~S}$ was marked as an abbreviation symbol "Cys" in the following section of this paper. All the reagents were of analytical grade and used as received without further purification.

In a typical fabrication procedure, $1 \mathrm{~g} \mathrm{CaCO}_{3}$ nanoparticles were added into 25 mL DEG solvent and sonicated for 15 min to form a template suspension. Meanwhile, $2 \mathrm{mM}$ thiourea or Cys was dissolved into another 25 mL DEG solvent under magnetic stirring, followed by the addition of $1 \mathrm{mM} \mathrm{SnCl}_{2}$ to obtain a homogeneous reaction solution. This solution was then poured into the $\mathrm{CaCO}_{3}$ template suspension mentioned above and stirred at room temperature for $10 \mathrm{~h}$ to acquire a new suspension, which was later transferred into a $60 \mathrm{~mL}$ Teflon-lined stainless steel autoclave and maintained at $150{ }^{\circ} \mathrm{C}$ for $24 \mathrm{~h}$, then cooled down to room temperature naturally. The resulting solid products were washed and collected by centrifuging several times with absolute ethanol. Subsequently, they were dried at $80^{\circ} \mathrm{C}$ for $6 \mathrm{~h}$ in a vacuum oven, and annealed at $400{ }^{\circ} \mathrm{C}$ for $3 \mathrm{~h}$ in a tube furnace with flowing $\mathrm{N}_{2}$ atmosphere to improve the crystallinity. After that, the products were etched by dilute hydrochloric acid and washed with distilled water to remove the $\mathrm{CaCO}_{3}$ templates, then dried at $60{ }^{\circ} \mathrm{C}$ overnight before characterization.

\subsection{Characterization}

The morphology and size of the raw $\mathrm{CaCO}_{3}$ template nanoparticles were examined by a scanning electron microscope (SEM, JSM-7600F, JEOL Ltd.). The size 
distribution of them in DEG solvent was measured with a laser particle analyzer (Zetasizer Nano ZS, Malvern Instrument Co.).

The morphology and hollow structure of the products were detected by the scanning electron microscope (SEM, JSM-7600F, JEOL Ltd.) equipped with a scanning transmission electron microscopy mode (STEM, SM-74240RTED, JEOL Ltd.). The phase and composition of them were analyzed with an X-ray diffractometer (XRD, D/Max 2500VB2+/PC, Rigaku Co., Ltd.), and an energy dispersive X-ray analyzer (EDX, JED-2300, JEOL Ltd.). The formation mechanism of the hollow structure was studied in terms of the affinity between reaction reagents and $\mathrm{CaCO}_{3}$ template in different reaction systems, by using a series of infrared absorption spectra recorded on a Fourier transform infrared spectrometer (FT/IR-6200, JASCO Co., Ltd.). Then, the nitrogen adsorption-desorption isotherm of the hollow structured product was measured at $77 \mathrm{k}$ with an adsorption analyzer (BEL SORP-max-NS, BEL Japan. Inc.), based on which the pore size distribution and specific surface area were calculated by Barret-Joyner-Halenda (BJH) method and Brunauer-Emmett-Teller (BET) method, respectively. At last, the optical properties of the hollow structured product were examined on a UV-VIS-NIR spectrophotometer (UV-3150, Shimadzu Co.). The corresponding absorption spectrum was recorded at room temperature after the product particles were dispersed in ethanol solution, with a concentration of around $6 \mu \mathrm{g} \cdot \mathrm{mL}^{-1}$.

\section{Results and discussion}

\subsection{Synthesis and characterization}

Prior to the synthesis of tin sulfide hollow structure, we first did SEM characterization to the raw $\mathrm{CaCO}_{3}$ template powder, which was directly adhered to the 
surface of a copper support for observation. As shown in Fig. 2, the obtained high magnification SEM image (bottom of inset) presents diversity in the geometric shape of individual $\mathrm{CaCO}_{3}$ nanoparticle, such as sphere, cube, wedge, and other irregular forms. The multiple shapes of nanoparticles exhibit non-uniform 3D sizes, most of which lie in the range of $60-120 \mathrm{~nm}$, and the average size of them is around $80 \mathrm{~nm}$ as provided by the manufacturer. Furthermore, the low magnification SEM image (top of inset) suggests that it is very easy for the $\mathrm{CaCO}_{3}$ nanoparticles to spontaneously aggregate together and stack into quantities of 3D hierarchical microclusters. The microclusters present a variety of irregular shapes with size ranging from 2 to $5 \mu \mathrm{m}$ in 3D directions. After being ultrasonically dispersed in DEG solvent for $15 \mathrm{~min}$, the sizes of them displayed a narrower distribution between 0.8 and $2.5 \mu \mathrm{m}$ centering at $1.6 \mu \mathrm{m}$, as confirmed by the light scattering analysis in Fig. 2. The results indicate that even undergoing an ultrasonication in DEG solvent, the $\mathrm{CaCO}_{3}$ nanoparticle powder still remained the state of microclusters only with a certain decrease in 3D sizes. To some extent, these microclusters should be loosely packed microstructures featuring numerous interior voids / pinholes available for mass transportation, since they have received an ultrasonic treatment and their stacking units (individual $\mathrm{CaCO}_{3}$ particle) possess non-uniform geometric shapes and 3D sizes. The interesting structural characteristics of $\mathrm{CaCO}_{3}$ nanoparticle powder in DEG solvent, together with its easily removable property by dilute acid, would promote it to be an ideal template material for the synthesis of novel hollow nanoparticle-based 3D microcluster-like tin sulfide products. Accordingly, we designed a facile route to the synthesis of the hollow structured target product, via an initial $\mathrm{CaCO}_{3}$ template-assisted solvothermal coating process in DEG solvent, followed by annealing treatment in $\mathrm{N}_{2}$ atmosphere to improve the product crystallinity and 
subsequent acid corrosion to remove the template cores. The solvothermal processes were performed in two separated $\mathrm{CaCO}_{3}$ suspension systems, where the same tin source but different sulfur sources (thiourea or Cys) were used for a comparison study.

Fig. 3 shows the morphology and interior characteristic of the product synthesized with thiourea as the sulfur source, which was observed after removal of $\mathrm{CaCO}_{3}$ template by acid treatment. From the typical SEM image in Fig. 3(a), it is seen that the product mainly comprises irregular-shaped agglomerates of highly compacted nanoparticles with no pinholes or cracks, accompanied by a few scattered small particles. The corresponding STEM image in Fig. 3(b) reveals their non-hollow but dense interiors, indicating a failure to the synthesis of hollow 3D tin sulfide microclusters in this case. It means the product nuclei undoubtedly did not undergo a coating growth process on the surface / intersurface of the 3D hierarchical $\mathrm{CaCO}_{3}$ microclusters, which thereby did not play a good template role as expected.

However, in contrast to the above case, the product synthesized with Cys as an alternative sulfur source was detected to display a very different morphology and interior structure. Fig. 4 shows its SEM and STEM images characterized after removal of $\mathrm{CaCO}_{3}$ template. Seen from the low magnification SEM image in Fig. 4(a), the product is composed of a large number of microclusters. The microclusters exhibit irregular shape, non-uniform size, and the size range is quite similar to the $\mathrm{CaCO}_{3}$ templates (agglomerates of nanoparticles) obtained after ultrasonic treatment in DEG solvent. Besides, the representative high magnification SEM and STEM images, presented in Fig. 4(b-d), reveal that every single microcluster is assembled by numerous hollow nanoparticles with an average shell thickness around $17 \mathrm{~nm}$. These hollow nanoparticles crosslink to each other and form a 3D hierarchical network-like 
microstructure. Most noticeably, the inner cavities of the hollow nanoparticles, in both the shape and size, seem to be good copies of the nanoparticles stacked in $\mathrm{CaCO}_{3}$ microcluster templates, perhaps implying a successful coating growth of the product nuclei surrounding the $\mathrm{CaCO}_{3}$ nanoparticle units.

For searching further evidences to the speculation of coating growth in the Cys-containing system, the product before removing $\mathrm{CaCO}_{3}$ microcluster template was, likewise, investigated by SEM and STEM characterizations for a comparison. The resulting images are presented in Fig. 5(a) and (b), respectively. As can be seen, it also composes irregular-shaped and non-uniform sized 3D microstructures assembled by plenty of nanoparticles with an average size of around $80 \mathrm{~nm}$, very similar to its counterpart after removal of $\mathrm{CaCO}_{3}$ template. However, its nanoparticle units do not possess hollow inner cavities; consequently only dense inherent structure could be observed. The distinct structural comparison above suggests that the nano-scale holes inside the network-like 3D hierarchitectures (shown in Fig. 4) were definitely generated after the removal of $\mathrm{CaCO}_{3}$ microcluster skeletons. It means the product nuclei in the Cys-containing system indeed underwent a solvothermal coating growth on the surface / intersurface of the 3D hierarchical $\mathrm{CaCO}_{3}$ microclusters, which therefore did play a good template role as expected. From XRD comparison of the $\mathrm{CaCO}_{3}$ template and solvothermal coating products before / after acid corrosion treatment (Fig. 5(c)), it is known that the template core can be totally removed by dilute hydrochloric acid and the obtained shell layer (i.e., the hollow 3D network-like microcluster product) belongs to pure orthorhombic SnS phase (JCPDS Card File, 65-3812). Using Scherrer's equation [19] and XRD data of the highest diffraction peak of the pure SnS shell layer, its average crystallite size was estimated to be around $8.4 \mathrm{~nm}$, nearly half of the shell thickness $(17 \mathrm{~nm})$, thereby indicating a 
two-layer coating growth of the SnS crystalline grains surrounding the microcluster-like $\mathrm{CaCO}_{3}$ template skeletons. Based on EDX analysis, the $\mathrm{Sn} / \mathrm{S}$ atomic ratio of the SnS crystalline grains is about 1 / 1.2, showing a little bit deviation from the stoichiometry $(1 / 1)$, which is a common phenomenon for semiconductors [20].

\subsection{Formation mechanism}

The aforesaid discussions reveal dramatic variations on both the morphology and inherent structure for the products synthesized in the two separated $\mathrm{CaCO}_{3}$ suspension systems, thiourea- and Cys-containing, respectively. Significantly, only in the Cys-containing system the SnS product nuclei could perform an effective solvothermal coating growth on the surface / intersurface of the 3D hierarchical $\mathrm{CaCO}_{3}$ microcluster templates and, as a result, we could successfully obtain the hollow nanoparticle-based SnS microclusters with 3D network hierarchitectures after the removal of $\mathrm{CaCO}_{3}$ skeletons by acid treatment. According to previous reports [21, 22], the most crucial factor in a coating process is the affinity between template and starting materials of coating layer. So, herein, it is supposed that the adhesion ability of the reaction reagents on $\mathrm{CaCO}_{3}$ templates might be insufficient in the thiourea-containing suspension system but much stronger in the Cys-containing system, thereby leading to an invalidation of the $\mathrm{CaCO}_{3}$ template in the former case while an excellent role play in the latter case. To verify this viewpoint, we examined the surface state of the $\mathrm{CaCO}_{3}$ templates collected from the above two different suspensions without solvothermal reaction yet (i.e., precursor suspensions), using FT-IR spectrum technique since it is an effective method for detecting trace amount of adsorbate and estimating adhesion ability $[23,24]$. Before the collection of $\mathrm{CaCO}_{3}$ 
templates, the two suspensions were first stirred at room temperature for $10 \mathrm{~h}$ to make sure a sufficient contact of the $\mathrm{CaCO}_{3}$ with the starting materials of SnS coating layer, then were washed with absolute ethanol and distilled water by centrifuging several times. Finally, two $\mathrm{CaCO}_{3}$ template samples were collected and dried at $60{ }^{\circ} \mathrm{C}$ overnight under vacuum condition before the FT-IR characterization. For supplying a reference, the FT-IR characterization was also performed on the raw $\mathrm{CaCO}_{3}$ template powder.

Fig. 6 presents the FT-IR spectral comparison of the three $\mathrm{CaCO}_{3}$ samples as illustrated above. Seen from Fig. 6(a), the raw $\mathrm{CaCO}_{3}$ template powder exhibits several groups of absorption bands in the wide region of $500-2700 \mathrm{~cm}^{-1}$, all of which could be assigned to the characteristic infrared absorptions of $\mathrm{CaCO}_{3}$ [25-27]. Besides, it displays a broad band from 3300 to $3500 \mathrm{~cm}^{-1}$ and two small peaks centering at around 2860 and $2970 \mathrm{~cm}^{-1}$. The former broad band probably relates to the stretching vibration of $\mathrm{O}-\mathrm{H}$ bond of water molecules adsorbed by the raw $\mathrm{CaCO}_{3}$ powder [27]. The latter two small peaks may arise from the adhesion of some residual rosin reagent, which was used during the preparation of raw $\mathrm{CaCO}_{3}$ powder according to the information provided by the Shiraishi Calcium Co., Ltd, and they possibly associate with the symmetric and asymmetric stretch mode of C-H bond of rosin, respectively $[28,29]$.

In comparison to the raw $\mathrm{CaCO}_{3}$ powder, the $\mathrm{CaCO}_{3}$ collected from the thiourea-containing precursor suspension exhibits a rather similar FT-IR spectrum as plotted by Fig. 6(b), revealing poor adhesion ability of the reaction reagents on $\mathrm{CaCO}_{3}$ templates in this suspension system. Whereas the $\mathrm{CaCO}_{3}$ collected from another precursor suspension, i.e. the Cys-containing system, presents some new features in the FT-IR spectrum. As depicted by Fig. 6(c), the new features originate 
from the strong peak between 1500 and $1750 \mathrm{~cm}^{-1}$, which displays a similar shape profile relative to the normal vibration peak of carbonate ion $\left(\mathrm{CO}_{3}{ }^{2-}\right.$, centering at 1540 $\mathrm{cm}^{-1}$ in the same wavenumber region of Fig. $\left.6(\mathrm{a})\right)$ of the raw $\mathrm{CaCO}_{3}$ powder $[25,27]$, but accompanied by a considerable enhancement in the intensity and a noticeable broadening in the peak width at half height as well as an identifiable shift of the peak center towards the new position (ca. $1580 \mathrm{~cm}^{-1}$ ). Such new features suggest that the surface state of the $\mathrm{CaCO}_{3}$ template collected from the Cys-containing precursor suspension was affected by the starting materials of SnS coating layer, and the effect was still detectable though the measured sample had been washed with ethanol and distilled water for several times. This point provides a powerful proof to the existence of strong affinity between the template and the reaction reagents in this suspension system. The strong affinity is deduced to most probably result from the interaction of the nanoparticles stacked in $\mathrm{CaCO}_{3}$ microcluster template and the carboxyl group (-COOH) contained in Cys reagent, since the active functional groups (mainly $\mathrm{Ca}^{2+}$ ) on the surface of nano- $\mathrm{CaCO}_{3}$ have been reported to be able to attach the $-\mathrm{COOH}$ group by forming carboxylate via ionic bond $[23,24]$. According to literatures [23, 24], the formed carboxylate usually shows a characteristic infrared absorption peak at around 1610 or $1597 \mathrm{~cm}^{-1}$, which is very close to the normal vibration peak of $\mathrm{CO}_{3}{ }^{2-}$ from $\mathrm{CaCO}_{3}$ template itself (centering at $1540 \mathrm{~cm}^{-1}$, as shown in Fig. 6(a)) [25, 27]. Therefore, it is supposed that they may well overlap each other and, as a result, contribute to the aforesaid new features of the strong peak (centering at $1580 \mathrm{~cm}^{-1}$ ) in the wavenumber region of $1500-1750 \mathrm{~cm}^{-1}$ in Fig. 6(c).

The above FT-IR analyses prove that the strong affinity between $\mathrm{CaCO}_{3}$ template and reaction reagents indeed only exists in the Cys-containing suspension system, which provides an essential factor for the successful solvothermal coating of SnS on 
$\mathrm{CaCO}_{3}$ in this system, while the failure in the other system (thiourea-containing suspension). During the coating process, it is deduced that the Cys-containing precursor solution could penetrate into the $\mathrm{CaCO}_{3}$ microcluster template through the voids / pinholes inside, and forward the coating growth of SnS nuclei on both the surface and intersurface of the template. So once removing the $\mathrm{CaCO}_{3}$ template skeleton by acid treatment, the hollow nanoparticle-based SnS microcluster product with network-like 3D hierarchitecture (as shown in Fig. 4) was obtained.

Now, in a conclusion, the successful fabrication of hollownanoparticle-based SnS microcluster product from Cys-containing system could be mainly ascribed to a combination of the following three reasons: i) microcluster-like stacking patterns of $\mathrm{CaCO}_{3}$ nanoparticles in DEG solvent despite of undergoing an ultrasonic dispersion, ii) strong adhesion of Cys on nano- $\mathrm{CaCO}_{3}$ through the possible ionic bond interaction between them, and iii) good removability of the $\mathrm{CaCO}_{3}$ template by dilute acid.

\subsection{Nitrogen sorption and UV-vis absorption measurements}

Besides the morphology, structure, composition and formation mechanism shown above, we further studied the specific surface area and porosity of the hollow nanoparticle-based SnS microcluster product by $\mathrm{N}_{2}$ sorption analysis and its optical properties by UV-vis absorption measurement, in order to acquire more information available for exploring potential applications of this novel 3D hierarchical tin sulfide product.

Fig. 7 shows the $\mathrm{N}_{2}$ adsorption-desorption isotherm of the product, which can be identified as IV type with a hysteresis loop characteristic of mesoporous material, according to the IUPAC classification [30, 31]. The total pore volume was evaluated to be ca. $0.066 \mathrm{~cm}^{3} \cdot \mathrm{g}^{-1}$ based on the single point adsorption at the relative pressure 
$\mathrm{P} / \mathrm{P}_{0}=0.99$. The corresponding $\mathrm{BJH}$ pore size distribution data (inset) calculated from the desorption branch of the isotherm suggest that the majority of pores possess an average size around $2.4 \mathrm{~nm}$, besides, some amount of pores are about $4.6 \mathrm{~nm}$. The two groups of mesopores presumably arise from the spaces among small nanocrystallites within the shells of hollow nanoparticle units of the microcluster product. Such porous nature provides the novel hollow nanoparticle-based and microcluster-like SnS 3D hierarchitectures with a 1 - 5 times higher BET specific surface area (ca. 87 $\mathrm{m}^{2} \cdot \mathrm{g}^{-1}$ ), in comparison to the previously reported 3D microflower samples which usually comprise plenty of nanopetals / nanosheets / nanorods but exhibit lower specific surface areas (e.g., 23.8, 47.3, 14.4, $\mathrm{m}^{2} \cdot \mathrm{g}^{-1}$ ) [2, 13, 32].

Fig. 8 (inset) shows the UV-vis absorption spectrum of the novel 3D hierarchical SnS microcluster product measured in ethanol solution. It displays a wide absorption from ultraviolet to visible light regions, similar to the photosensitive area of the previously reported 3D SnS microflowers (built by nanorods or nanobelts [33]) dispersed in ethanol solution, too. However, in the whole absorption region, it shows a long and sloping tail, very different from the absorption feature of those reported microflowers (nearly horizontal in longer-wavelength visible light region [33]). The long and sloping absorption tail of the hollow SnS microcluster product here is similar to the case of hollow CuS and ZnS particles discussed in literatures [34] and [35], which have attributed the predominant forming reason of such tail to light scattering effect of the hollow particles in dispersion system. Since this kind of interesting tail didn't appear in the absorption spectrum of those 3D SnS microflowers reported in literature [33], it is deduced that their light scattering ability might be very weak in comparison to the novel 3D SnS microcluster product obtained in our work. The much strong light scattering property of our microcluster product is probably due to 
the following two reasons: i) it comprises numerous hollow nanoparticle units and possesses much high specific surface area, thus can provide a large shell interface on both the outer and inner surfaces which are always available to scatter light [36]; ii) the spherical inner cavities of its hollow nanoparticle units are beneficial for producing additional light multi-scattering effect [37]. According to literature [37], such hollow-structure induced enhancement on the scattering effect could improve the product's light absorption ability in the UV-vis region. Fig. 8 also shows the $(a \text { h } v)^{2}$ vs. (hv) curve of the hollow SnS microcluster product, plotted depending on its absorption spectrum and the well-known equation $(\alpha h v)^{2}=B\left(h v-E_{\mathrm{g}}\right)$, where $\alpha, h v, E_{\mathrm{g}}$ and $B$ are absorption coefficient, photon energy, band gap and a constant, respectively $[33,38]$. Obviously, the curve tends to a straight line in the high photon energy region. By extrapolating the straight line to the $\mathrm{h} v$-axis $[33,38]$, the band gap $E_{\mathrm{g}}$ of the hollow microcluster product was estimated to be about $1.8 \mathrm{eV}$, showing blue shifts relative to the values $(1.3-1.4 \mathrm{eV})$ of 3D SnS microflowers described in the above literature [33]. The blue-shift phenomenon could be mainly ascribed to the quantum confinement effect [35], since the average shell thickness (around $17 \mathrm{~nm}$ ) of hollow nanoparticle units inside the 3D microcluster product is much smaller than the thickness / diameter (100 - $200 \mathrm{~nm})$ of nanobelts / nanorods assembled in the previously reported microflowers [33]. Anyhow, the SnS microcluster product here as against the previous microflowers is considered to have a unique hollow structural advantage that can produce scattering-enhanced absorption in the whole UV-vis light region.

The analyses above reveal that the hollow nanoparticle-based SnS microcluster product as a novel 3D hierarchical tin sulfide sample possesses three apparent advantages including very larger specific surface area, richer mesopore structure and 
scattering-enhanced absorption in UV-vis light region, compared to the previously reported 3D hierarchical microflowers built by nanosheets, nanorods, nanobelts, etc. The former two advantages would provide the new SnS 3D hierarchitecture sample with much more active reaction positions and communicable channels for reactant molecules and resultants, assuming to use it in the fields like lithium-ion batterys [2, 39], photocatalysts [15, 40] and gas sensors [41], where usually require quantities of active positions and effective mass transportation. While, the latter one advantage would give the new SnS 3D hierarchitecture sample better performance, if apply it in some optical fields (e.g. photocatalysts [15, 40] and solar cells [40, 42]) which always ask wide and heavy light absorption as much as possible for high-efficiency energy conversion. Furthermore, the new SnS 3D hierarchitecture sample owns a unique structural characteristic. It contains numerous microcluster-like stacked hollow nanoparticles with rich mesopores inside the shells, probably being beneficial to the capsulation, storage, and controlled release of drugs or other materials. Therefore, the hollow nanoparticle-based SnS product with microcluster-like 3D network hierarchitectures in this work should have much more extensive and promising applications, as against those previously reported microflower-like 3D hierarchitectures assembled by nanopetals, nanosheets, nanorods, nanobelts, etc. For the detailed application study, we decide to carry out in next research plans.

\section{Conclusions}

A novel hollow nanoparticle-based SnS crystal product was fabricated via a template-assisted solvothermal coating route with $\mathrm{CaCO}_{3}$ nanoparticles as the template materials and DEG as the solvent medium, followed by annealing treatment in $\mathrm{N}_{2}$ atmosphere and subsequent corrosion of template by dilute acid. For a 
comparison study, the solvothermal coating of $\mathrm{SnS}$ on $\mathrm{CaCO}_{3}$ template was performed in two separated systems, where the same tin source but different sulfur sources (thiourea and Cys) were used, respectively. Results show that successful coating only occurred in the Cys-containing system, mainly due to the strong adhesion of Cys on nano- $\mathrm{CaCO}_{3}$ through the possible ionic bond interaction between them. The obtained SnS coating product was found to comprise many microcluster-like 3D network hierarchitectures assembled by hollow nanoparticles, which exhibit an average shell thickness of $17 \mathrm{~nm}$. Such hollow nanoparticle-based SnS hierarchitectures were considered to have well copied the outlines of the microcluster-like stacking patterns of $\mathrm{CaCO}_{3}$ nanoparticles in DEG solvent. This novel SnS product owns several apparent advantages compared to those previously reported micro-scaled tin sulfide 3D hierarchitectures, for instance, very larger specific surface area, unique hollow nanoparticle units with rich mesopores inside the shells, and scattering-enhanced absorption in UV-vis light region. Thus, it is believed to have much more extensive and promising applications in the fields of lithium-ion batteries, photocatalysts, gas sensors, solar cells, capsulation and delivery. For the detailed application study, we are considering doing in next research plans.

\section{Acknowledgment}

This work was supported by the Advanced Low Carbon Technology Research and Development Program (ALCA) of the Japan Science and Technology Agency (JST).

\section{References}

[1] C.R. Patra, A. Odani, V.G. Pol, D. Aurbach, A. Gedanken, Microwave-assisted 
synthesis of tin sulfide nanoflakes and their electrochemical performance as Li-inserting materials, Journal of Solid State Electrochemistry, 11 (2007) 186 194.

[2] J. Zai, X. Qian, K. Wang, C. Yu, L. Tao, Y. Xiao, J. Chen, 3D-hierarchical SnS 2 micro / nano-structures: controlled synthesis, formation mechanism and lithium ion storage performances, CrystEngComm, 14 (2012) 1364 - 1375.

[3] B. Chen, X. Xu, F. Wang, J. Liu, J. Ji, Electrochemical preparation and characterization of three-dimensional nanostructured $\mathrm{Sn}_{2} \mathrm{~S}_{3}$ semiconductor films with nanorod network, Materials Letters, 65 (2011) 400 - 402.

[4] S.C. Ray, M.K. Karanjai, D. DasGupta, Structure and photoconductive properties of dip-deposited $\mathrm{SnS}$ and $\mathrm{SnS}_{2}$ thin films and their conversion to tin dioxide by annealing in air, Thin Solid Films, 350 (1999) 72 - 78.

[5] F. Lu, J. Yang, R. Li, N. Huo, Y. Li, Z. Wei, J. Li, Gas-dependent photoresponse of SnS nanoparticles-based photodetectors, Journal of Materials Chemistry C, 3 (2015) 1397 - 1402.

[6] P. Tang, H. Chen, F. Cao, G. Pan, K. Wang, M. Xu, Y. Tong, Nanoparticulate SnS as an efficient photocatalyst under visible-light irradiation, Materials Letters, 65 (2011) $450-452$.

[7] H. Karami, S. Babaei, Application of tin sulfide-tin dioxide nanocomposite as oxygen gas-sensing agent, International Journal of Electrochemical Science, 8 (2013) 12078 - 12087.

[8] A.M. Tripathi, S. Mitra, Tin sulfide (SnS) nanorods: structural, optical and lithium storage property study, RSC Advances, 4 (2014) 10358 - 10366.

[9] Z. Zhang, C. Shao, X. Li, Y. Sun, M. Zhang, J. Mu, P. Zhang, Z. Guo, Y. Liu, Hierarchical assembly of ultrathin hexagonal $\mathrm{SnS}_{2}$ nanosheets onto electrospun 
$\mathrm{TiO}_{2}$ nanofibers: enhanced photocatalytic activity based on photoinduced interfacial charge transfer, Nanoscale, 5 (2013) 606 - 618.

[10] H. Ke, W. Luo, G. Cheng, X. Tian, Z. Pi, Synthesis of flower-like $\mathrm{SnS}_{2}$ nanostructured microspheres using PEG 200 as solvent, Micro \& Nano Letters, 4 (2009) 177 - 180.

[11] W. Cai, J. Hu, Y. Zhao, H. Yang, J. Wang, W. Xiang, Synthesis and characterization of nanoplate-based SnS microflowers via a simple solvothermal process with biomolecule assistance, Advanced Powder Technology, 23 (2012) $850-854$.

[12] S. Biswas, S. Kar, S. Chaudhuri, Thioglycolic acid (TGA) assisted hydrothermal synthesis of SnS nanorods and nanosheets, Applied Surface Science, 253 (2007) 9259 - 9266.

[13] Q. Wu, L. Jiao, J. Du, J. Yang, L. Guo, Y. Liu, Y. Wang, H. Yuan, One-pot synthesis of three-dimensional $\mathrm{SnS}_{2}$ hierarchitectures as anode material for lithium-ion batteries, Journal of Power Sources, 239 (2013) 89 - 93.

[14] H. Hu, B. Yang, J. Zeng, Y. Qian, Morphology evolution of SnS nanocrystals: from 3D urchin-like architectures to 1D nanostructures, Materials Chemistry and Physics, 86 (2004) 233 - 237.

[15] H. Liu, Y. Su, P. Chen, Y. Wang, Microwave-assisted solvothermal synthesis of 3D carnation-like $\mathrm{SnS}_{2}$ nanostructures with high visible light photocatalytic activity, Journal of Molecular Catalysis A: Chemical, 378 (2013) 285 - 292.

[16] S. Biswas, S. Kar, T. Ghoshal, S. Chaudhuri, Fabrication of $\mathrm{SnS}_{2}$ flower like nanoflake assemblies through thermal evaporation, Journal of Nanoscience and Nanotechnology, 7 (2007) 4540 - 4545.

[17] M. Fuji, C. Takai, R.R. Virtudazo, Development of new templating approach for 
hollow nanoparticles and their applications, Advanced Powder Technology, 25 (2014) 91 - 100.

[18] B. Zhao, G. Shao, B. Fan, Y. Xie, B. Sun, R. Zhang, Preparation and microwave absorption of porous hollow $\mathrm{ZnO}$ by $\mathrm{CO}_{2}$ soft-template, Advanced Powder Technology, 25 (2014) 1761 - 1766.

[19] B.D. Cullity, S.R. Stock, Elements of X-ray Diffraction, 3rd ed., Prentice Hall, Englewood Cliffs NJ, 2001.

[20] X. Xu, F. Wang, J. Liu, Z. Li, J. Ji, J. Chen, Structural and optical studies of ZnS nanocrystal films prepared by sulfosalicylic acid $\left(\mathrm{C}_{7} \mathrm{H}_{6} \mathrm{O}_{6} \mathrm{~S}\right)$-assisted galvanostatic deposition with subsequent annealing, Thin Solid Films, 520 (2012) 6864 - 6868.

[21] M.L. Breen, A.D. Dinsmore, R.H. Pink, S.B. Qadri, B.R. Ratna, Sonochemically produced ZnS-coated polystyrene core-shell particles for use in photonic crystals, Langmuir, 17 (2001) 903 - 907.

[22] C. Takai, M. Fuji, K. Fujimoto, Skeletal silica Nanoparticles prepared by control of reaction polarity, Chemistry Letters, 40 (2011) 1346 - 1348.

[23] B. Shentu, J. Li, Z. Weng, Effect of oleic acid-modified nano-CaCO 3 on the crystallization behavior and mechanical properties of polypropylene, Chinese Journal of Chemical Engineering, 14 (2006) 814 - 818.

[24] H.A. Al-Hosney, S. Carlos-Cuellar, J. Baltrusaitis, V.H. Grassian, Heterogeneous uptake and reactivity of formic acid on calcium carbonate particles: a Knudsen cell reactor, FTIR and SEM study, Physical Chemistry Chemical Physics, 7 (2005) 3587 - 3595.

[25] F.A. Andersen, L. Brecevic, Infrared spectra of amorphous and crystalline calcium carbonate, Acta Chemica Scandinavica, 45 (1991) 1018 - 1024.

[26] M.A. Legodi, D. de Waal, J.H. Potgieter, S.S. Potgieter, Rapid determination of 
$\mathrm{CaCO}_{3}$ in mixtures utilizing FT-IR spectroscopy, Minerals Engineering, 14 (2001) $1107-1111$.

[27] L. Brecevic, D. Kralj, On calcium carbonates: from fundamental research to application, Croatica Chemica Acta, 80 (2007) 467 - 484.

[28] A. Yoshihara, T. Maeda, Y. Imai, Spectroscopic characterization of ambers and amber-like materials, Vibrational Spectroscopy, 50 (2009) 250 - 256.

[29] A.B. Sifontes, B. Gutierrez, A. Monaco, A. Yanez, Y. Diaz, F.J. Mendez, L. Llovera, E. Canizales, J.L. Brito, Preparation of functionalized porous nano-g- $\mathrm{Al}_{2} \mathrm{O}_{3}$ powders employing colophony extract, Biotechnology Reports, 4 (2014) 21 - 29.

[30] H.F. Shao, X.F. Qian, Z.K. Zhu, The synthesis of ZnS hollow nanospheres with nanoporous shell, Journal of Solid State Chemistry, 178 (2005) 3522 - 3528.

[31] C. Deng, H. Hu, X. Ge, C. Han, D. Zhao, G. Shao, One-pot sonochemical fabrication of hierarchical hollow $\mathrm{CuO}$ submicrospheres, Ultrasonics Sonochemistry, 18 (2011) 932 - 937.

[32] M. He, L.X. Yuan, Y.H. Huang, Acetylene black incorporated three-dimensional porous $\mathrm{SnS}_{2}$ nanoflowers with high performance for lithium storage, RSC Advances, 3 (2013) 3374 - 3383.

[33] Q. Han, M. Wang, J. Zhu, X. Wu, L. Lu, X. Wang, Great influence of a small amount of capping agents on the morphology of SnS particles using xanthate as precursor, Journal of Alloys and Compounds, 509 (2011) 2180 - 2185.

[34] X. Yu, C. Cao, H. Zhu, Q. Li, C. Liu, Q. Gong, Nanometer-sized copper sulfide hollow spheres with strong optical-limiting properties, Advanced Functional Materials, 17 (2007) 1397 - 1401.

[35] Y. Ma, L. Qi, J. Ma, H. Cheng, Facile Synthesis of Hollow ZnS Nanospheres in 
Block Copolymer Solutions, Langmuir, 19 (2003) 4040 - 4042.

[36] K. Nelson, Y. Deng, Enhanced light scattering from hollow polycrystalline $\mathrm{TiO}_{2}$ particles in a cellulose matrix, Langmuir, 24 (2008) 975 - 982.

[37] M.C. Huang, T.H. Wang, C.C. Wu, W.S. Chang, J.C. Lin, T.H. Yen, The optical, structural and photoelectrochemical characteristics of porous hematite hollow spheres prepared by DC magnetron sputtering process via polystyrene spheres template, Ceramics International, 40 (2014) 10537 - 10544.

[38] Y.C. Zhang, Z.N. Du, K.W. Li, M. Zhang, Size-controlled hydrothermal synthesis of $\mathrm{SnS}_{2}$ nanoparticles with high performance in visible light-driven photocatalytic degradation of aqueous methyl orange, Separation and Purification Technology, $81(2011) 101-107$.

[39] D.D. Vaughn II, O.D. Hentz, S. Chen, D. Wang, R.E. Schaak, Formation of SnS nanoflowers for lithium ion batteries, Chemical Communications, 48 (2012) 5608 $-5610$.

[40] J. Chao, Z. Xie, X. Duan, Y. Dong, Z. Wang, J. Xu, B. Liang, B. Shan, J. Ye, D. Chen, G. Shen, Visible-light-driven photocatalytic and photoelectrochemical properties of porous $\mathrm{SnS}_{\mathrm{x}}(\mathrm{x}=1,2)$ architectures, CrystEngComm, 14 (2012) $3163-3168$.

[41] W. Shi, L. Huo, H. Wang, H. Zhang, J. Yang, P. Wei, Hydrothermal growth and gas sensing property of flower-shaped $\mathrm{SnS}_{2}$ nanostructures, Nanotechnology, 17 (2006) 2918 - 2924.

[42] D.J. Lewis, P. Kevin, O. Bakr, C.A. Muryn, M.A. Malik, P. O’Brien, Routes to tin chalcogenide materials as thin films or nanoparticles: a potentially important class of semiconductor for sustainable solar energy conversion, Inorganic Chemistry Frontiers, 1 (2014) 577 - 598. 


\section{Figure captions:}

Fig.1. Molecular structures of (a) thiourea and (b) Cys.

Fig.2. SEM images (inset) of the raw $\mathrm{CaCO}_{3}$ particles and their size distribution in DEG solvent after sonication for $15 \mathrm{~min}$.

Fig.3. Morphology and interior characteristic of the product synthesized with thiourea as the sulfur source and observed after removing $\mathrm{CaCO}_{3}$ template, (a) a typical SEM image and (b) its corresponding STEM image.

Fig.4. Morphology and interior characteristic of the product synthesized with Cys as the sulfur source and observed after removing $\mathrm{CaCO}_{3}$ template, (a) a low magnification SEM image, (b, c) a high magnification SEM and its corresponding STEM image, (d) a much higher magnification STEM image.

Fig.5. Morphology and structure of the product synthesized with Cys as the sulfur source: $(a, b)$ a magnified SEM and its corresponding STEM images measured before removing $\mathrm{CaCO}_{3}$ template; (c) the XRD comparison of $1 \mathrm{CaCO}_{3}$ template and 2 / 3 solvothermal coating products before / after corrosion treatment by dilute hydrochloric acid.

Fig.6. FT-IR spectra of (a) the raw $\mathrm{CaCO}_{3}$ powder and the $\mathrm{CaCO}_{3}$ collected from (b) thiourea-containing and (c) Cys-containing precursor suspensions.

Fig.7. Nitrogen adsorption-desorption isotherm of the hollow product synthesized with Cys as the sulfur source (inset: pore size distribution curve).

Fig.8. $(a \mathrm{~h} v)^{2}$ vs. (hv) curve and the corresponding UV-vis absorption spectrum (inset) of the hollow product synthesized with Cys as the sulfur source.

\section{Figures:}




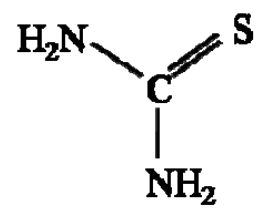

(a)

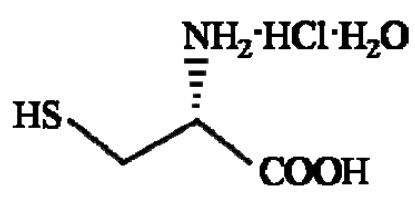

(b)

Fig.1.

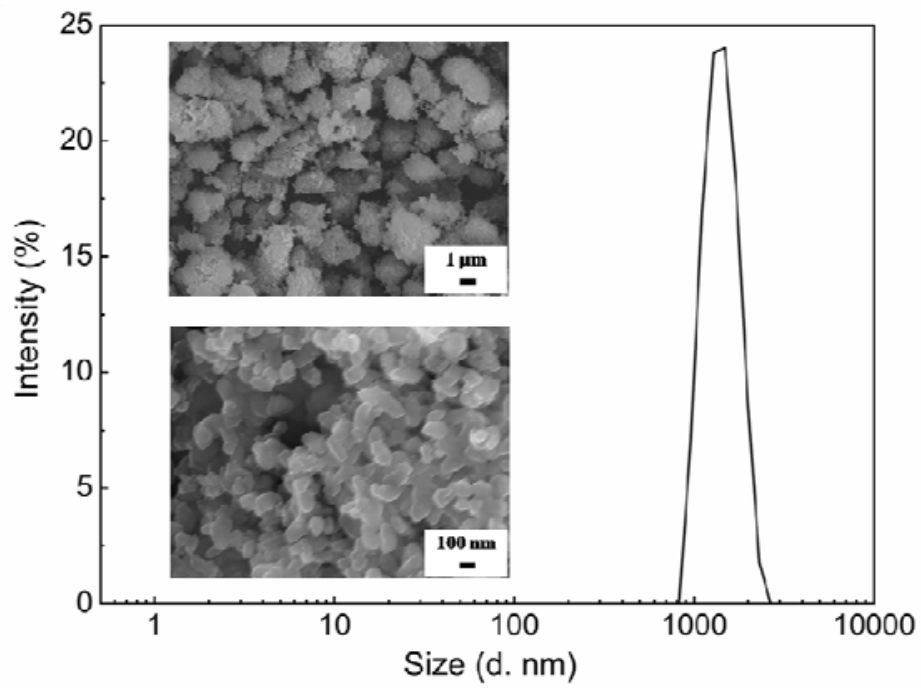

Fig.2. 


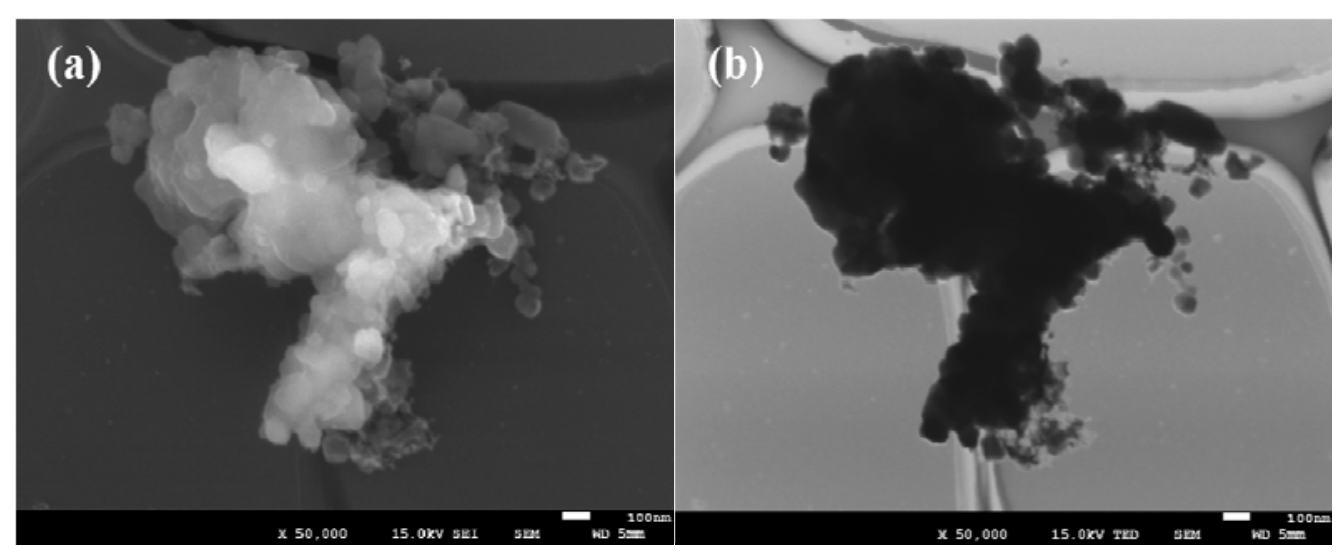

Fig.3.
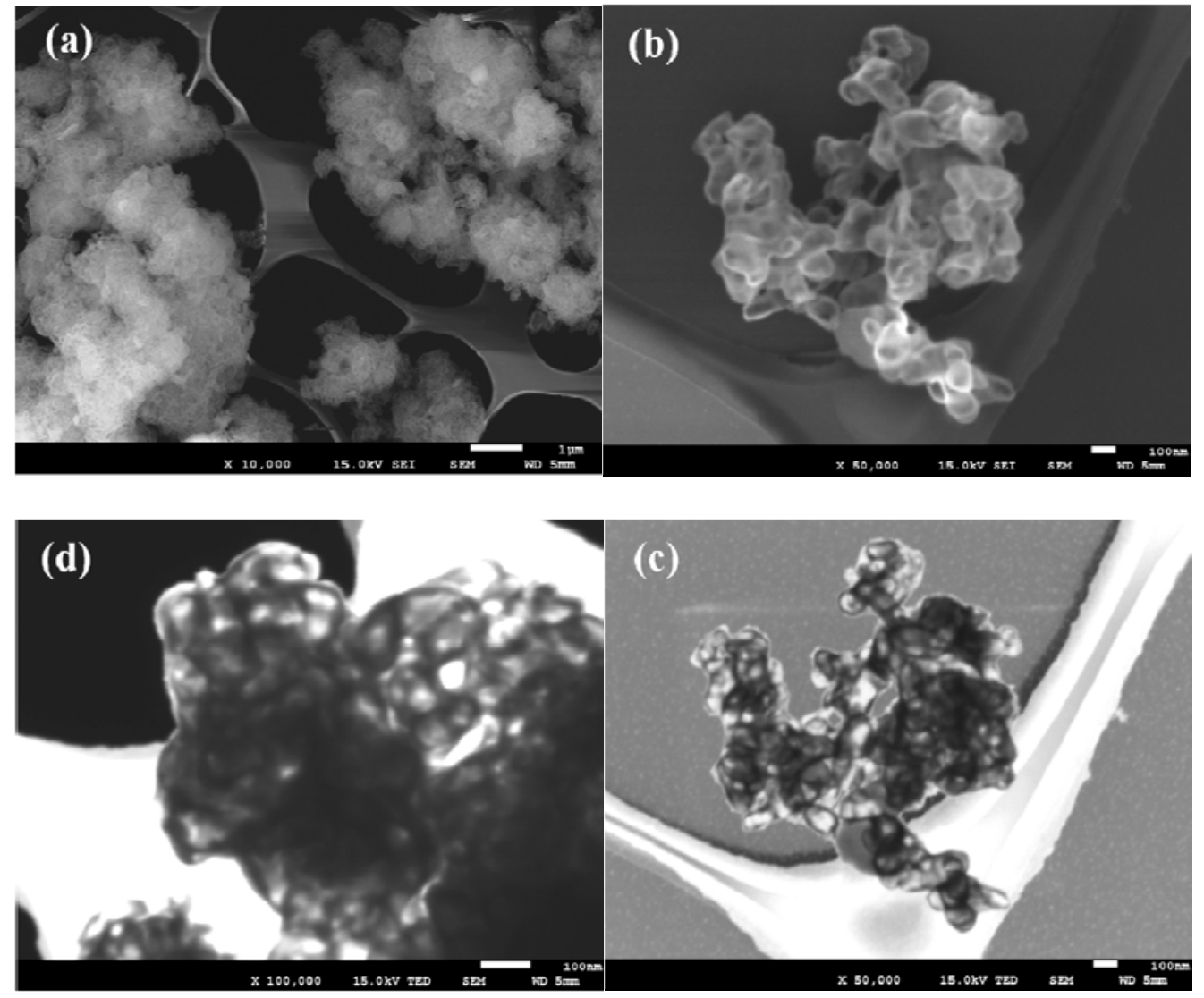

Fig.4. 

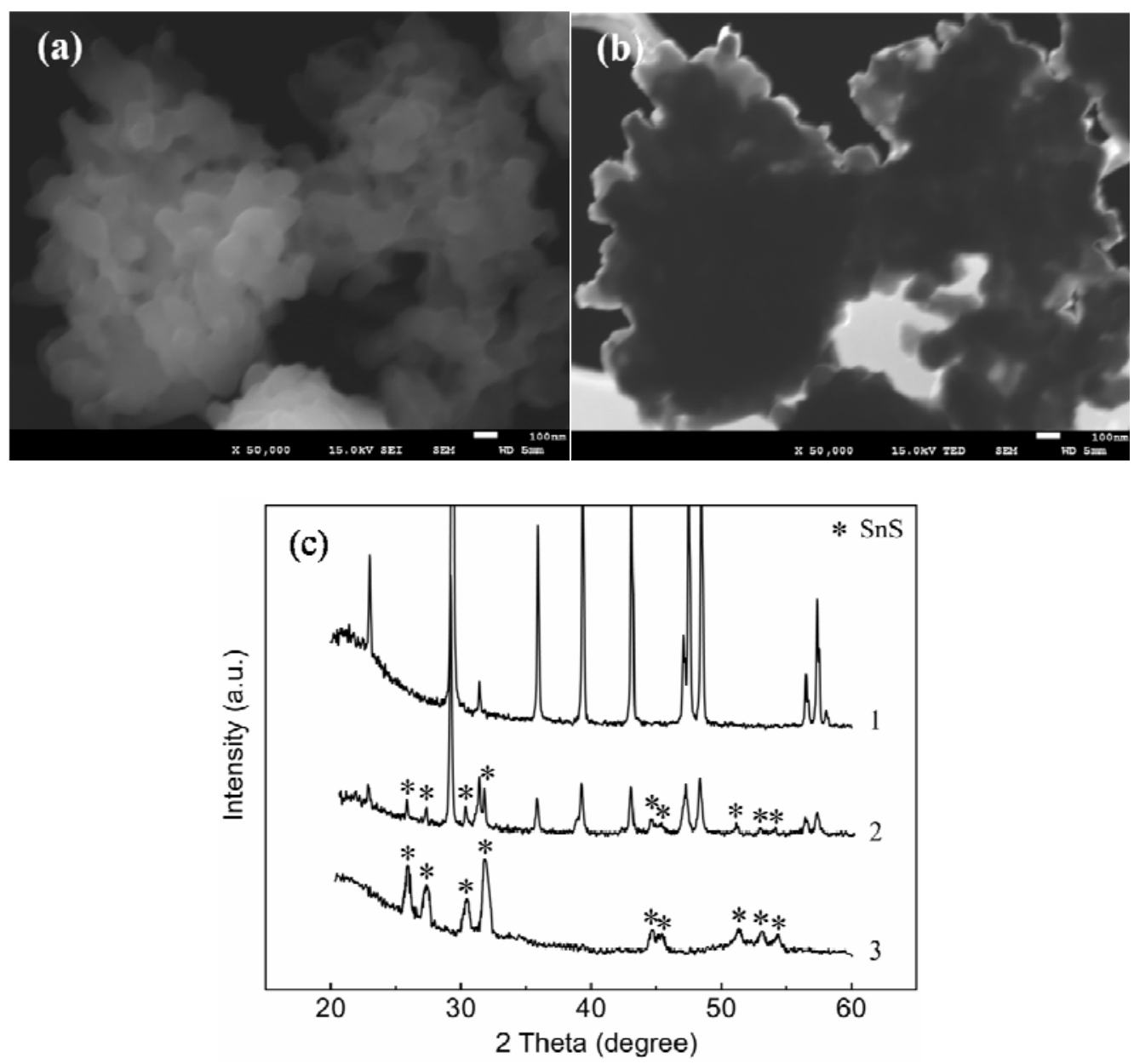

Fig.5.

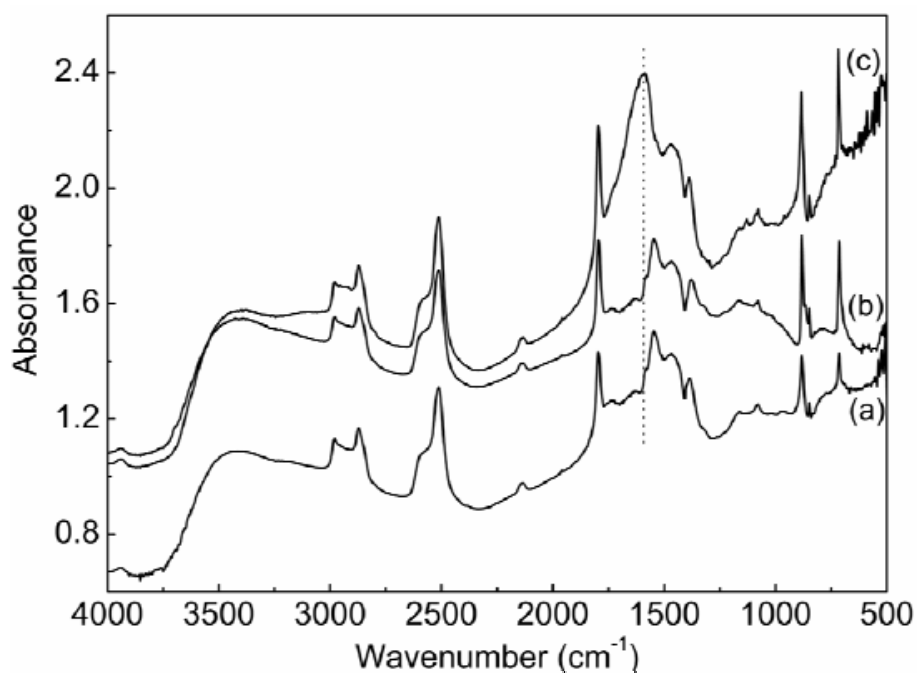

Fig.6. 


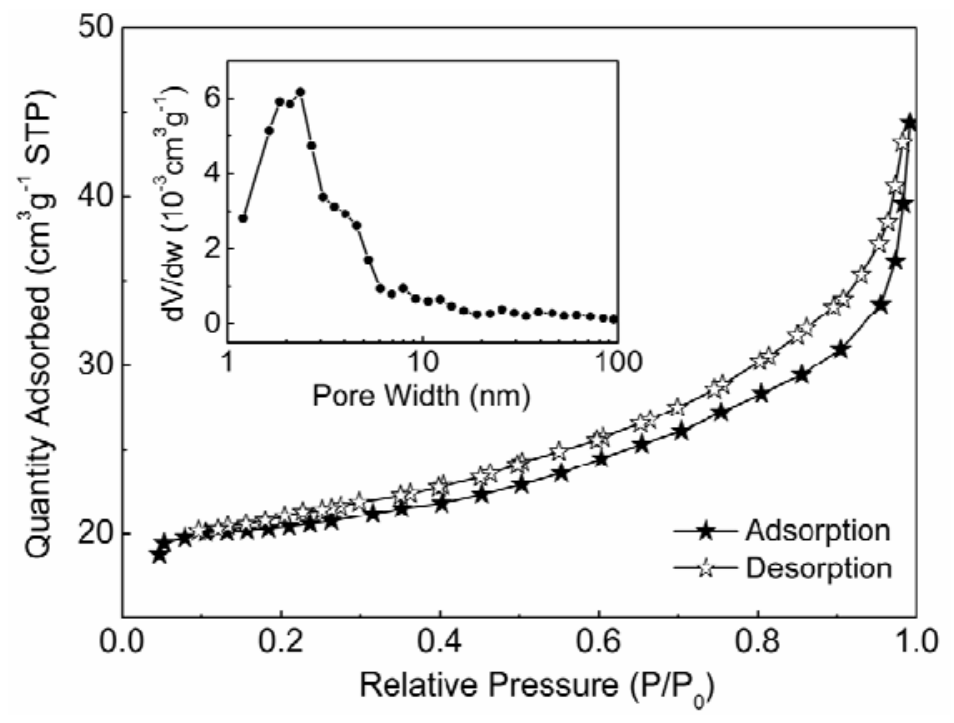

Fig.7.

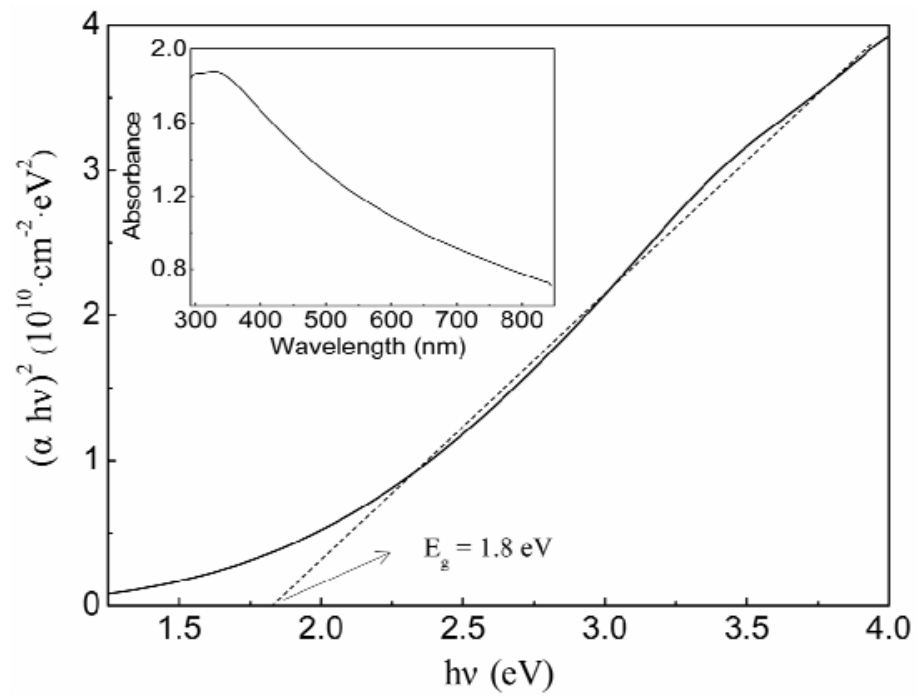

Fig.8. 